\title{
Magnetic Nanoparticles for Tunable Microwave Metamaterials
}

\author{
Natalia Noginova ${ }^{1}$, Quincy Leon Williams ${ }^{1}$, Panagiotis Dallas ${ }^{2}$, Emmanuel P. Giannelis ${ }^{2}$ \\ ${ }^{1}$ Center for Materials Research Norfolk State University, Norfolk VA 23504 \\ ${ }^{2}$ KAUST-CU Center for Energy and Sustainability Cornell University, Ithaca NY 14853
}

\begin{abstract}
Commonly, metamaterials are electrically engineered systems with optimized spatial arrangement of subwavelength sized metal and dielectric components. We explore alternative methods based on use of magnetic inclusions, such as magnetic nanoparticles, which can allow permeability of a composite to be tuned from negative to positive at the range of magnetic resonance. To better understand effects of particle size and magnetization dynamics, we performed electron magnetic resonance study on several varieties of magnetic nanoparticles and determined potential of nanoparticle use as building blocks for tunable microwave metamaterials.
\end{abstract}

\section{INTRODUCTION}

Recent progress in metamaterias has inspired scientists and engineers who have proposed numerous exciting applications of artificial materials with custom tailored electric permittivity $\varepsilon$ and magnetic permeability $\mu$ in radio frequency and optical ranges. Revolutionary basic science ideas and unparalleled applications have been proposed for double negative (both $\varepsilon$ and $\mu$ are negative), single negative $(\varepsilon \mu<1)$, near-zero $\varepsilon$, and near-zero $\mu$ materials, as well as the metamaterials with strong anisotropy of $\varepsilon$ and $\mu$ [1-5].

Starting from the dawn of the era of metamaterials, researchers exploited electrical engineering approach to metamaterial design, based on the optimized spatial arrangement of sub-wavelength sized metal and passive dielectric components. Unfortunately, such devices typically operate only in narrow frequency ranges; include significant losses; have relatively large sizes of the building blocks ("meta-atoms"); and are difficult to produce on an industrial scale.

Current research trends espouse approaches based on active control of $\varepsilon$ and $\mu$ to tune the materials' parameters, and bottom up fabrication routes to allow for relatively inexpensive manufacturing [6,7]. In our work we explore composites with magnetic nanosize inclusions in a dielectric matrix as promising systems for active microwave metamaterials with tunable permeability.

The use of natural magnetic materials in as a constituent part of metamaterials is attractive as it provides high values of magnetic permeability, as well as a possibility to tune material parameters with the external magnetic fields $[8,9]$. In the vicinity of a ferromagnetic resonance (at microwave range), real part of permeability, $\mu$ ', can vary in a broad range (from positive to negative values depending on a particular material), while $\mu$ " demonstrates an absorption line $[9,10]$. The frequency of magnetic resonance can be controlled by external magnetic fields providing possibility of tuning. Feasibility of this approach to negative refraction index materials was shown in [10], based on bulk ferromagnetic materials. Composite structures filled with superparamagnetic nanoparticles can provide various advantages, including lesser eddy currents losses as well as easier fabrication routes [7].

In this paper, we investigate magnetization dynamics of small $(<50 \mathrm{~nm})$ iron oxide nanoparticles as a function of their size. We also estimate the potential for their use in tunable metamaterials.

Metamaterials: Fundamentals and Applications V, edited by Allan D. Boardman

Nader Engheta, Mikhail A. Noginov, Nikolay I. Zheludev, Proc. of SPIE Vol. 8455, 845531

(C) 2012 SPIE · CCC code: 0277-786/12/\$18 - doi: 10.1117/12.931755 


\section{EXPERIMENTAL TECHNIQUES AND RESULTS}

Series of ferrofluid samples with particles of a different size were fabricated by chemical synthesis or obtained from Nanomaterials \& Nanofabrication Laboratories (NNLabs) and OceanNanotech (Table 1). Magnetization data for NNLabs samples were obtained from manufacturer specifications. Characterization of Ocean Nanotech samples was performed in house with a SQUID magnetometer. All samples could be dissolved in a nonpolar solvent.

Table 1. List of ferrofluid systems

\begin{tabular}{|c|l|l|l|l|c|}
\hline Size & Manufacturer & Particle & Surfactant & Solvent & $\begin{array}{c}\text { Magnetization after solvent } \\
\text { evaporation } \\
(\mathrm{T}=300 \mathrm{~K}, \mathrm{H}=4500 \mathrm{Oe})\end{array}$ \\
\hline $5 \pm 1 \mathrm{~nm}$ & NNLabs & $\mathrm{Fe}_{3} \mathrm{O}_{4}$ & Oleic Acid & Toluene & $30 \mathrm{emu} / \mathrm{g}$ or higher \\
\hline $9 \pm 1 \mathrm{~nm}$ & OceanNanotech & $\mathrm{Fe}_{3} \mathrm{O}_{4}$ & Oleic Acid & Chloroform & $45 \mathrm{emu} / \mathrm{g}$ \\
\hline $11 \pm 1 \mathrm{~nm}$ & OceanNanotech & $\mathrm{Fe}_{3} \mathrm{O}_{4}$ & Oleic Acid & Chloroform & $26 \mathrm{emu} / \mathrm{g}$ \\
\hline $15 \pm 2 \mathrm{~nm}$ & Cornell & $\mathrm{Au}_{\mathrm{Fe}} \mathrm{O}_{3}$ & Oleylamine & Toluene & $\mathrm{N} / \mathrm{A}$ \\
\hline $20 \pm 3 \mathrm{~nm}$ & NNLabs & $\mathrm{Fe}_{3} \mathrm{O}_{4}$ & Oleic Acid & Toluene & $20 \mathrm{emu} / \mathrm{g}$ \\
\hline $30 \pm 3 \mathrm{~nm}$ & NNLabs & $\mathrm{Fe}_{3} \mathrm{O}_{4}$ & Oleic Acid & Toluene & $10 \mathrm{emu} / \mathrm{g} @$ \\
\hline $40 \pm 5 \mathrm{~nm}$ & OceanNanotech & $\mathrm{Fe}_{3} \mathrm{O}_{4}$ & Oleic Acid & Toluene & N/A \\
\hline
\end{tabular}

Electron Magnetic Resonance (EMR) studies were performed at room temperature using a standard Bruker EMX spectrometer operating at the microwave frequency of $10 \mathrm{GHz}$. The EMR signal (the derivative of microwave absorption) was recorded in the dependence on the static magnetic field, which was slowly swept from $0 \mathrm{G}$ to 7000 G. The modulation frequency was $100 \mathrm{kHz}$ and amplitude was $3 \mathrm{G}$.

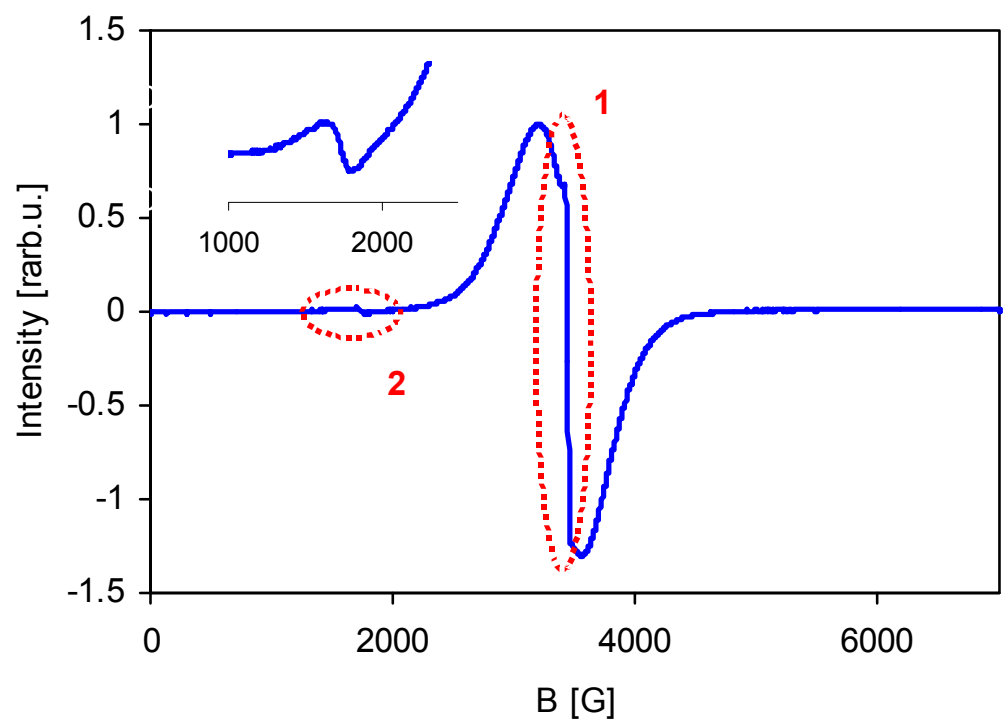

Fig. 1. Typical EMR signal in the well-diluted ferrofluid. The narrow signal at the resonance frequency (1) and half-field signal (2 and inset) are indicated. 
In the first series of experiments, the EMR signals were recorded in well-diluted systems in order to characterize an individual behavior of particles. The samples of ferrofluids were diluted to the point, where the shape of the EMR line was not affected by further dilution. Fig. 1 shows a typical EMR signal observed in nanoparticles with the nominal size of $9 \mathrm{~nm}$. As was discussed in Refs. [11, 12], the EMR in nanoparticles can be described using "quantization" approach, considering resonance transitions between energy levels of a giant spin which corresponds to the total magnetic moment of a nanoparticle [11, 13]. A narrow feature (Trace 1) with Lande factor $(\mathrm{g}) \sim 2$ observed in the middle of a broader resonance line, is an indication of discrete energy levels. Another interesting feature is low-field signals observed at $\mathrm{H}_{0} / \mathrm{n}$, where $\mathrm{H}_{0}$ is the resonance field and $\mathrm{n}=1,2,3 \ldots$ The low-field signals can be explained in the frames of the quantization model as well. They were ascribed to multiple quantum transitions originated from effects of magnetic anisotropy and dipolar interactions of nanoparticles. The both features are common in small quantum systems, such as single electron spins or small spin clusters [14], and not expected in classical macroscopic ferromagnets.

Our experiments with particles of a different size provided an opportunity to observe the evolution of quantum features with increase in the particle size. Fig. 2a shows the EMR signals obtained in nanoparticles of various sizes. Each trace was normalized to the double integral under the curve, which is proportional to the static magnetization ("amount of magnetic material") in the corresponding sample. The sharpest resonance signals were obtained with the smallest diameter particles. As the diameter of the nanoparticles approached $40 \mathrm{~nm}$, the main absorption line became very broad and shifted toward the lower fields. The resonance peak at half-field and a narrow feature at resonance were clearly observed in samples with diameter $<20 \mathrm{~nm}$. While the position of the narrow line did not change $\left(\mathrm{H}_{0}=3425 \mathrm{G}\right)$, its magnitude quickly decreased upon increase in the particle size. The changes in the relative magnitude of the narrow feature are clearly seen in Fig. 2 b, whish shows the derivative of the EMR signal. Detailed analysis of the nanoparticle magnetization dynamics and its evolution with increase in nanoparticle size will be published elsewhere.

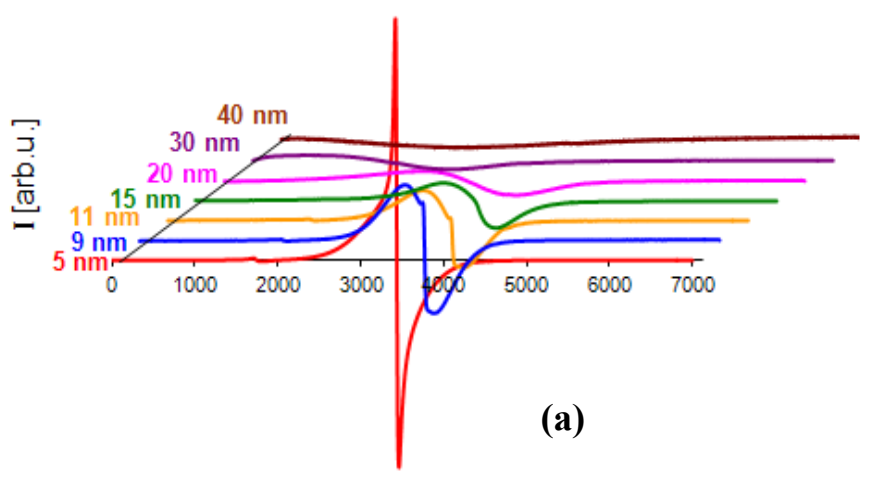

$\mathrm{B}[\mathrm{G}]$

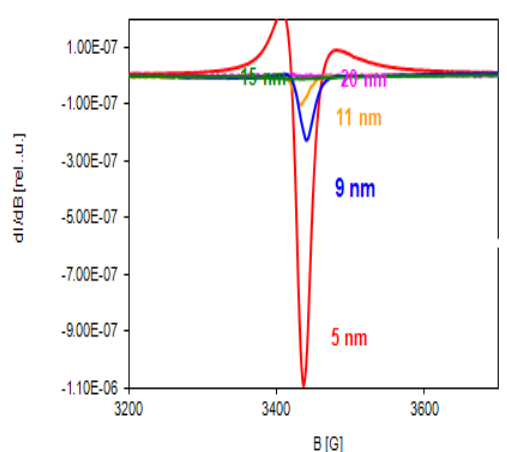

$B[G]$

Figure 2. (a) EMR signal in ferrofluids.. (b) Derivative of EMR signal (second derivative of absorption.)

Let us now discuss opportunities, which nanoparticles can provide for the design of tunable metamaterials. First, let us note that magnetic properties of the superparamagnetic particles differ from those of ferromagnetic materials. Due to relatively small size and increasing role of the thermal fluctuations, the magnetization $\mathrm{M}_{0}$ in superparamagnetic systems is lower than that in classical ferromagnetic materials, but still is relatively high. It depends on temperature and external magnetic field, following the Langevin law [15] and saturates in high fields or at lower temperatures to the ferromagnetic value. 
For tuning the resonant transverse magnetic permeability $\mu=\mu^{\prime}-\mathrm{i} \mu$ " in a broad range (such as tuning $\mu$ ' from positive to negative values), the higher $\mathrm{M}_{0}$, the better. Another important parameter is the width of the EMR line, which should be relatively narrow. According to Fig. 2, narrow EMRs were observed in smallest nanoparticles 5 and $9 \mathrm{~nm}$. Taking into account the magnetization data (Table 1), they are the most promising candidates.

However, due to moderate magnetic properties of iron oxides, high concentrations of particles might be necessary to achieve high enough volume magnetization. On the other hand, high densities of magnetic nanoparticles could result in a significantly broadened EMR due to strong dipolar interactions, which is unwanted effect.

To test the effects of broadening and estimate a possible tuning range, we studied systems with an extremely high concentration of particles. Dried samples on strips of thin tissue paper were produced from the ferrofluids by simple evaporation of the solvent.
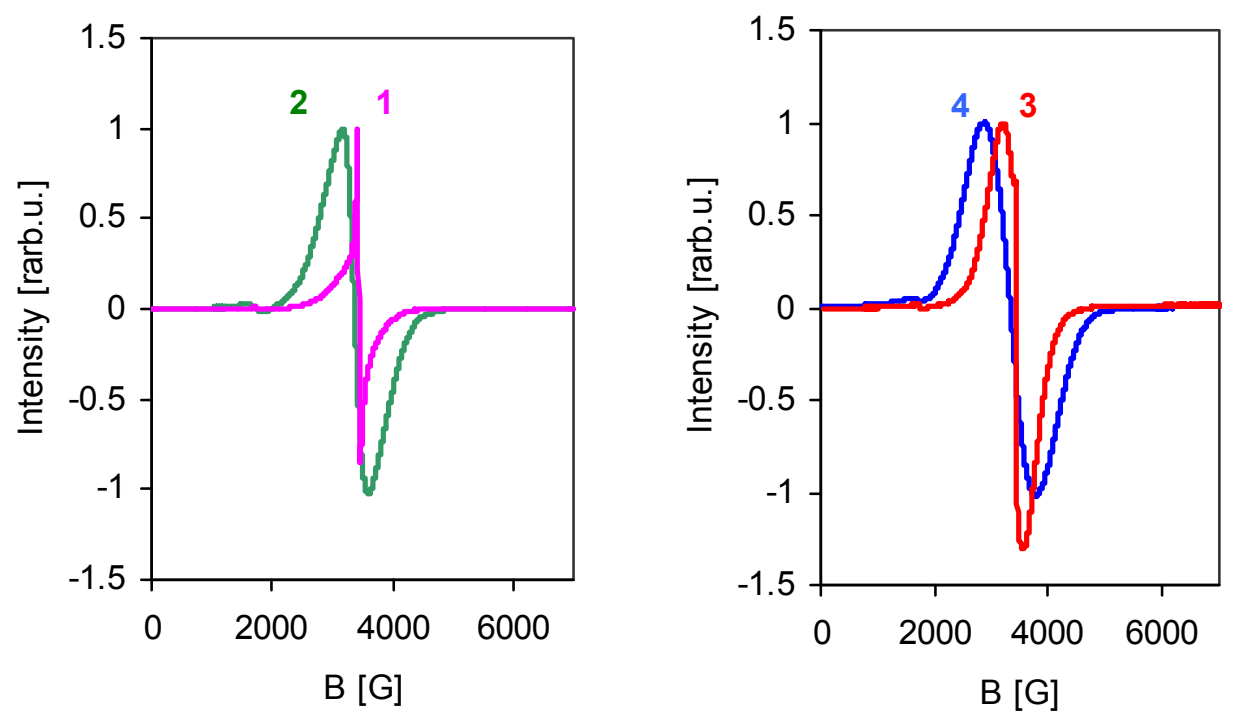

Fig. 3. EMR in diluted (Traces 1 and 3) and dried samples (2 and 4) with $5 \mathrm{~nm}$ nanoparticles (a) and $9 \mathrm{~nm}$ nanoparticles (b). The signals are normalized to maximum

Fig. 3 shows the EMR in well-diluted liquid systems (Traces 1 and 3) and corresponding dried samples (2 and 4) containing $5 \mathrm{~nm}$ (Fig. 3 a) and $9 \mathrm{~nm}$ (Fig. 3b) nanoparticles. Broadening was observed in the dried systems in comparison with liquids, however, the EMR line was still relatively narrow. The half-widths were estimated as 360 Oe in $5 \mathrm{~nm}$ and 550 Oe in $9 \mathrm{~nm}$ samples correspondingly.

\section{Discussion}

The transverse microwave magnetic permeability can be found as

$\mu=\mu^{\prime}-i \mu^{\prime \prime}=1+4 \pi\left(\chi^{\prime}-i \chi^{\prime \prime}\right)$ 
where $\chi^{\prime}(\mathrm{x})$ and $\chi^{\prime \prime}(\mathrm{x})$ are real and imaginary parts of the magnetic susceptibility. If the lineshape is Lorentzian, $\chi^{\prime}(\mathrm{x})$ and $\chi^{\prime}(\mathrm{x})$ are [15]

$$
\chi^{\prime}=\frac{M_{0}}{2 \delta} \frac{x / \delta}{1+(x / \delta)^{2}} \quad \text { and } \quad \chi^{\prime}=\frac{M_{0}}{2 \delta} \frac{1}{1+(x / \delta)^{2}}
$$

where $x=H-H_{0} \quad \mathrm{H}_{0}$ and $\delta$ are the center field and half-width of the resonant absorption line. In our case, the lines are close to but not strictly Lorentzian. Instead, we will use the relation,

$$
\chi^{\prime}(x)=k I(x)_{\exp } x / \delta \quad \text { and } \quad\left(\chi^{\prime \prime}(x)=k I(x)_{\exp }\right.
$$

where $I(x)_{\text {exp }}$ is the experimental lineshape of the absorption line (integrated EMR signal), and $\mathrm{k}$ is the scaling factor, which can be estimated from magnetization data (Table 1) and relation between $\mathrm{M}_{0}$ and the area, $\mathrm{S}$, under the absorption line, $S=(\pi / 2) * M_{0}$.

$k=(\pi / 2) M_{0} / S$

Taking into account the content of surfactant of $\sim 25 \%$ [16], we estimated the magnetization per volume as $M_{0}=$ $120 \mathrm{emu} / \mathrm{cm}^{3}$ and $180 \mathrm{emu} / \mathrm{cm}^{3}$ for $5 \mathrm{~nm}$ and $9 \mathrm{~nm}$ samples correspondingly. Calculated values of $\mu$ are shown in Fig. 4. As one can see, in the both cases, the real part of permeability varies from -0.8 to 2 , in the dependence on the magnetic field. The line for the $9 \mathrm{~nm}$ sample is broader than in the $5 \mathrm{~nm}$ system, however, due to the higher value of the volume magnetization, the magnitude of the variation is approximately the same.
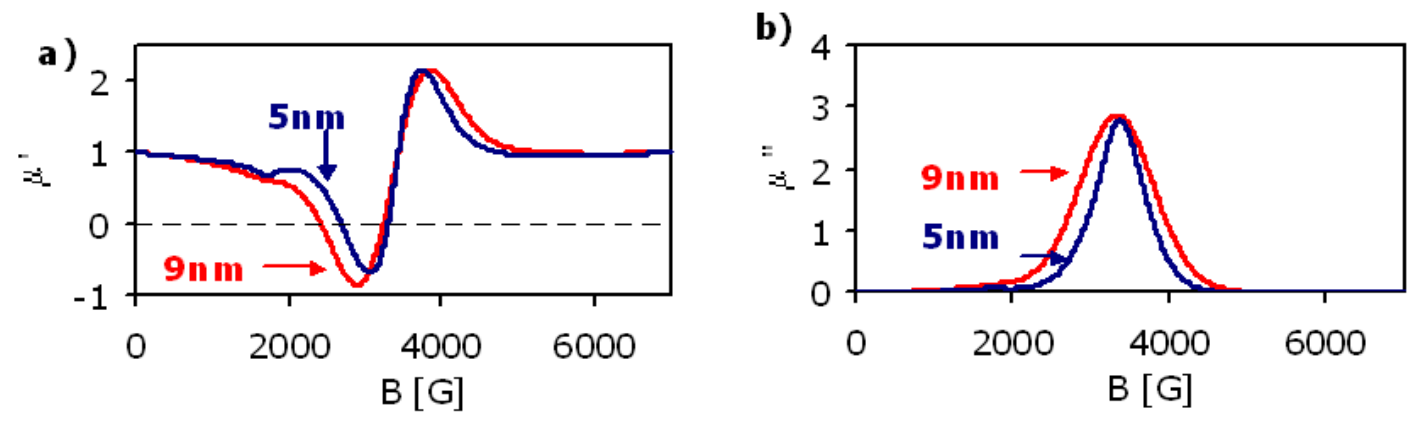

Fig. 4. Estimations of real (a) and imaginary (b) parts of permeability in dried samples with $5 \mathrm{~nm}$ and $9 \mathrm{~nm}$ particles (as indicated)

Note, that this range of tunability was obtained in samples with a very high density of the nanoparticles: nothing else but nanoparticles plus surfactants. For metamaterials applications, it would be necessary to include additional parts to design the electric permittivity. This could substantially decrease the volume magnetization. To solve this problem, the use of nanoparticles with higher magnetization is important, such as permalloy and iron platinum nanoparticles.

In conclusion, electron magnetic resonance was studied in iron oxide nanoparticles of various sizes. Evolution of the EMR signal upon increase in the particle size was observed, including broadening of the main resonance and sharp decrease in relative magnitude of "quantal" features. Microwave permeability was estimated in solid systems of $5 \mathrm{~nm}$ and $9 \mathrm{~nm}$ nanoparticles. It was shown that the real part of permeability can be tuned with the external magnetic field in the range from -0.8 to 2 . 


\section{Acknowledgments:}

This work was supported by following grants: NSF META-PREM \# DMR 1205457, AFOSR \# FA9550-09-1-0456, NSF IGERT \# DGE-0966188, UTC \# 10-567-001502C4. Authors thank V. A. Atsarkin for helpful discussions.

\section{References}

[1] J. B. Pendry, "Negative refraction makes a perfect lens". Phys. Rev. Lett. 85, 3966-3969 (2000).

[2] D.R. Smith, D.C. Vier, N. Kroll, and S. Schultz, "Direct calculation of permeability and permittivity for a left-handed metamaterial" Appl. Phys. Letters 77, 2246 (2000).

[3] D. Schurig, J. J. Mock, B. J. Justice, S. A. Cummer, J. B. Pendry, A. F. Starr and D. R. Smith, "Metamaterial electromagnetic cloak at microwave frequencies" Science 314, 977 (2006).

[4] Z. Jacob, L. V. Alekseyev and E. Narimanov, "Optical hyperlens: Far-field beyond the diffraction limit, “ Opt. Express 14, 8247 (2006).

[5] A. Alu and N. Engheta, "Guided Modes in a Waveguide Filled with a Pair of Single-Negative (SNG), Double-Negative (DNG), and/or Double-Positive (DPS) Layers" IEEE Transactions on Microwave Theory and Techniques, MTT-52, 199 (2004).

[6] C. Mitsumata, S. Tomaita, M. Hagiwara, and K. Akamatsu, "Electron magnetic resonance in interacting ferromagnetic-metal nanoparticle systems: experiment and numerical simulation" J. Phys.:Condens. Matter 22, 1 (2010).

[7] C. Morales, J. Dewdney, S. Pal, S.Skidmore, K. Stojak, H. Srikanth, T. Weller, and J. Wang, "Tunable magneto-dielectric polymer nanocomposites for microwave applications" IEEE Transactions on Microwave Theory and Techniques 59, 302 (2011).

[8] P. M. T. Ikonen, K. N. Rozanov, A. V. Osipov, P. Alitalo, and S. A. Tretyakov. "Magnetodielecric substrates in antenna miniaturization: potential and limitation" IEEE Transactions on Antennas and Propagation 54, No 11, 3391 (2006).

[9] A. L. Adenot-Engelvin, C. Dudek, P. Toneguzzo, O. Acher. "Microwave properties of ferromagnetic composites and metamaterials" J. of Eur. Cer. Soc., 27, 1029 (2007).

[10] Y. Poo, R. Wu, G. He, P. Chen, J. Xu, R. Chen, "Experimental verification of a tunable left-handed material by bias magnetic fields" Appl. Phy. Lett. 96, 161902 (2010).

[11] N. Noginova, F. Chen, T. Weaver, E. Giannelis, A. Burlinos, and V.A. Atsarkin. "Magnetic resonance in nanoparticles: between ferro- and paramagnetism". Journal of Physics: Condensed Matter, 19, 246208 (2007).

[12] N. Noginova, T. Weaver, E.P. Giannelis, A.B. Bourlinos, V. A. Atsarkin, V. V. Demidov. "Observation of multiple quantum transitions in magnetic nanoparticles". Phys. Rev. B 77, 014403 (2008).

[13] D. A. Garanin. “ Relaxation of superparamagnetic spins: Classical vs large-spin description ". Phys. Rev. B 78, 144413 (2008).

[14] D. Gatteschi, M. Fittipaldi, C. Sangregorio, L. Sorace. "Exploring the No-Man's Land between Molecular Nanomagnets and Magnetic Nanoparticles". Angewandte Chemie Int. Edit. 51, 4792 (2012).

[15] A. G. Gurevich and G. A. Melkov. "Magnetization Oscillations and Waves". CRC-Press, 1996. $464 p$.

[16] L. Zhang, R. He, H.-Ch. Gu "Oleic acid coating on the monodisperse magnetite nanoparticles". Appl. Surf. Science 253, 2611(2006). 